\title{
Uwi ni Sifa ya Ujumi wa Kiafrika? Mifano kutoka Diwani ya Mnyampala, Mashairi ya Saadan na Kivuli cha Mvumo
}

\author{
Birigitha Ngwano John \\ Idara ya Kiswahili, Chuo Kikuu cha Dodoma
}

\section{IKISIRI}

Uwi ni dhana ya kiujumi inayowatatiza watu wengi kwa sababu huko nyuma ujumi ulijulikana kama uzuri. Jamii mbalimbali ulimwenguni zina aina mbalimbali za ujumi. Afrika ina aina mahsusi za ujumi ambazo hutambulishwa na vipengele anuwai katika sanaa zake. Makala hii inaangazia uthibitisho wa kuwa uwi ni sifa ya kiujumi wa Waafrika. Aidha, data zilizotumika katika makala hii zimekusanywa kwa njia ya maktaba iliyohusisha usomaji wa vitabu, makala na tasnifu. Njia hii ilitumika kwa sababu ya kupata data za upili na kubaini mapengo yaliyojitokeza katika tafiti zilizotangulia. Vitabu teuli ni vya mashairi kutoka diwani tatu ambazo ni Diwani ya Mnyampala ya Mathias Mnyampala, Mashairi ya Saadan ya Saadan Kandoro na Kivuli cha Mvumo ya Rashid Mfaume yamehusishwa kama rejea kuthibitisha kuwa uwi ni sifa ya ujumi wa Waafrika. Nadharia ya Unegritudi inayokitwa katika mikabala ya Kiafrika, ndiyo iliyoongoza mjadala wa matokeo wa makala hii. Imethibitika kuwa uwi ni kitu cha kiujumi hivyo kinapaswa kumakinikiwa kwa namna iliyo sawa na uzuri.

\author{
Historia ya \\ Makala \\ Kupokelewa 15 \\ Aprili 2018
}

Kukubaliwa: 1

Disemba 2018

Istilahi Muhimu

Ujumi; Uwi;

Waafrika; Idili

\section{Jinsi ya kurejea makala hii:}

John, B.N. 2018. Uwi ni Sifa ya Ujumi wa Kiafrika? Mifano kutoka Diwani ya Mnyampala, Mashairi ya Saadan na Kivuli cha Mvumo. Mkwawa Journal of Education and Development, 2, 20-33. $\quad$ DOI: https://doi.org/10.37759/mjed.2018.2.1.2 


\subsection{Utangulizi}

Kipengele hiki kinafafanua istilahi muhimu katika makala haya ambazo ni; ujumi, ujumi wa Kiafrika na uwi. Halikadhalika, sehemu hii inafafanua Nadharia ya Unegritudi ambayo imetumika kama kiunzi cha mjadala wa matokeo ya makala.

\subsection{Dhana ya Ujumi}

Ujumi ni tafsiri ya neno la Kiingereza 'aesthetics' lililotumiwa kwa mara ya kwanza na Alexander Gottlieb Baumgarten mwaka 1735, (Ponera, 2014). Mtandao wa Dictionary.com, unafafanua dhana ya ujumi kama ifuatavyo:

Aesthetic is the branch of philosophy dealing with such notions as the beautiful, the ugly, the subline, the comic, etc. as applicable to the fine arts, with a view to establishing the meaning and validity of critical judgements concerning works of art, and the principles underling or justifying such judgements.

Ujumi ni tawi la falsafa linaloshughulika na dhana ya uzuri, ubaya, utukufu, ufutuhi, kama linavyohusika katika uchoraji likiwa na maoni ya kuimarisha uhalali wa maamuzi ya kiuhakiki kuhusu sanaa na kanuni zake (Tafsiri yangu).

Maelezo haya yanaonesha kuwa ujumi hujihusisha na uzuri pamoja na uwi kwa kuzingatia kanuni kadhaa. Kwa mujibu wa Concise Oxford English Dictionary (2001) katika Badru (2015:07), kanuni za ujumi zimerejelewa kama ifuatavyo:

A set of principles concerned with the nature and appreciation of beauty, especially in art. The branch of philosophy which deals with questions of beauty and artistic taste.

Fungu la kanuni zinazohusika na asili na uhakiki wa uzuri, hasa katika sanaa. Ni tawi la falsafa ambalo hujihusisha na maswali yanayohusu uzuri na vionjo vya sanaa. (Tafsiri yangu).

Kwa ujumla, ujumi ni taaluma inayoshughulikia uzuri, ubaya, ladha, ubora, udhalili na mambo yanayofanana na hayo. Pia, taaluma hii hujenga sheria, kanuni na taratibu zinazotumika katika kupima ubora wa kazi za sanaa. Taaluma hii ina vipengele vikuu vinne ambavyo ni uzuri/ ubaya katika asili, uzuri/ ubaya katika mtu, uzuri/ubaya katika dunia ya vitu na uzuri/ubaya katika sanaa.

\subsection{Dhana ya Ujumi wa Kiafrika}

Dhana ya ujumi wa Kiafrika imetokana na mazingira ya kukandamizwa kwa Mwafrika katika nyanja zote za maisha (Simchimba, 2012). Ni upinzani dhidi ya hujuma 

zilizofanywa na wakoloni dhidi ya heshima yake (Wafula na Njogu, 2003). Hivyo, Mwafrika anataka utamaduni wake utambulike na kutathiminiwa na watu kama zilivyo tamaduni zenye asili ya Kimagharibi.

Zirimu (1971) anashadidia kwamba jamii mbalimbali zina aina mbalimbali za ujumi. Mawazo ya Zirimu yanaashiria kuwa fasihi ya Kiafrika ina ujumi mahsusi ambao uhitaji kuhakikiwa kwa mikabala ya Kiafrika ili kuweza kueleweka vema. Hii inaukilia kuwa kuna ujumi unaotambulisha Afrika nzima. Makumba (2007), Temple (1959) na Oruka (1991) wanashadidia kwa kueleza kuwa Waafrika wana falsafa na ujumi wao ambao huwatambulisha. Hii ni kwa sababu kama Waafrika kama binadamu wengine ulimwenguni wana uwezo wa kuishi kulingana na misingi inayotawala jamii zao. Laswai (2015) anafafanua dhana ya ujumi wa Kiafrika kwa kusema:

Hii ni dhana inayojumuisha uzuri wote wa kiujumi utokanao na jamii, pamoja na mazingira ya ki-Afrika. Hutofautiana na jamii au mazingira mamoja na mengine ndani ya bara moja (Afrika) kutokana na tajriba na idili za jamii husika. Ujumi huu upo katika sanaa, hususan katika masimulizi ya kifasihi, na kwa Afrika uliitwa ujumi mweusi (Black Aesthetics) kama ulivyobainishwa na Zirimu (2007) (uk.12)

Nukuu tajwa hapo juu inaonesha kuwa kila jamii ina ujumi wake ambao hutofautiana na jamii nyingine. Ujumi wa Kiafrika ni uzuri, uwi, uchanya na uhasi unaohusisha mitazamo au jumla ya mawazo, yanayoakisi maisha ya Mwafrika ambayo yanatawaliwa na misingi mahsusi ya jamii za Kiafrika.

Ujumi wa Kiafrika wakati mwingine huitwa ujumi mweusi (Black Aesthetics) ambayo ni dhana inayojigeza katika utambuzi au falsafa ya uzuri, hususan katika sanaa na pia katika asili (Laswai, 2015). Tunakubaliana na mawazo haya kwa sababu falsafa kuu ya ujumi mweusi ni kwamba Waafrika nao ni watu walio na maisha yao, sanaa yao na taratibu zao za maisha ambazo hazitegemei kukamilishwa na jamii kutoka nje ya Afrika. Moja ya jukumu la ujumi wa Kiafrika ni kupigania utambuzi wa utu na maisha ya Mwafrika, ikiwemo sanaa. Utambuzi wa utu wa Mwafrika ulipiganiwa kwa namna nyingi na mbinu mbalimbali ikiwa ni pamoja na kutumia, kazi mbalimbali za kifasihi.

\subsection{Dhana ya Uwi}

Uwi ni kinyume cha uzuri na kutokuwepo kwa uwiano baina ya uhalisia wa kitu hicho na idili za jamii husika, (TUKI, 2013). Kwa hiyo, uwi ni matokeo ya kutokukubalika au kutokuwepo kwa uwiano wa uhalisia wa kitu fulani katika utamaduni fulani. Kwa 

ujumla, dhana ya uwi wa kazi ya fasihi na kama kipengele cha ujumi ni tata ambayo huwezi kuiamua kwa maneno ya moja kwa moja kwani hutofautiana kutoka mtu hadi mtu jamii hadi jamii. Hali kadhalika, dhana ya uwi wa kazi ya fasihi hutegemea pia hisia za mtu, juu ya kazi husika, kwani vipo au zipo zionekanavyo kuwa mbaya kwa watu fulani na ili hali kwa watu wengine zinaonekana kuwa nzuri. Hivyo basi, uwi huamliwa na mtu au watu husika kwa kuzingatia kaida za maisha yao, taratibu za nchi na misingi mbalimbali ya imani. Katika muktadha wa taaluma ya ujumi, uwi unapaswa uangaliwe kwa namna ya kuona uthamani ndani yake.

Hata hivyo, uzuri wa umbo la kazi ya fasihi si kipengele pekee ambacho huutambulisha ujumi wa Kiafrika, kwani vipo vipengele vingine anuai ambavyo hudhihirika katika kazi ya fasihi ambavyo huutambulisha ujumi wa Kiafrika. Vipengele hivyo ni kama: Imani za dini za jadi, umoja, ushababi, utii na adabu pamoja na ukarimu.

\subsection{Dhana ya Idili}

Idili ni ari, shauku, bidii, jitihada, kasi, hima au utashi wa mtu kufanya kazi (TUKI, 2014). Jamii ndiyo huamua uzuri/ ubaya wa kitu. Idili ni kigezo cha kiujumi ambacho katika muktadha wa fasihi huchukuliwa kuwa ndiyo nguzo ya msingi au kigezo ambacho jamii hujigeza kwacho, kama kipimo cha kiuhakiki au kulinganisha uzuri au ubaya wa jambo. Darwin (1882) ambaye ni mchunguzi aliyeishi Marekani kwa miaka mingi, katika kuwaongelea wanawake alisema; mwanamke mzuri ni mwenye paji fupi, kidevu kikubwa na kipana, mkao mzuri wa pua, rangi ya kahawia na matiti yanayoning’inia kuelekea chini. Hii inaonesha kuwa kwa kutumia kigezo cha idili, Darwin ameweza kupata namna yake ya kuona uzuri kwa mwanamke suala ambalo linaweza kuwa tofauti na namna mtu mwingine atakavyoweza kuuona. Luhwago (2013) anasema kuwa, jambo kubwa la msingi linalotakiwa kuzingatiwa ni kuwa makini wakati wa kutumia neno uwi/uzuri ni kuzingatia idili za jamii husika. Ikiwa uzuri/ uwi utatazamwa kwa kutumia kigezo cha desturi, hapo utathaminiwa kuwa upo chini ya ethos (ethics) kwa maana ya utamaduni, na ikiwa uzuri/ uwi utatazamwa kwa kuzingatia maneno au lugha kwa ujumla, utatathiminiwa kuwa upo chini ya Logos (Words). Kwa mujibu wa makala hii, idili imetumika kama kigezo cha kuutazama uwi ambao ni sifa ya ujumi wa Kiafrika kwa kuzingatia utamaduni wa jamii husika. 


\subsection{Nadharia ya Unegritudi}

Wamitila (2002) anasema Nadharia ya Unegritudi ilianzishwa miaka ya 1930. Mushengyezi (2003) anaeleza kwamba vuguvugu la Unegritudi ni zao la fikra za mshairi maarufu wa visiwa vya Caribbean aliyejulikana kwa jina la Aime Cessaire pamoja na raisi wa zamani wa Senegal, Leopard Sedar Senghor. Fikra hizi za Kinegritudi zilikuwa maarufu sana upande wa Afrika ya Magharibi. Watu waliokuwa mstari wa mbele katika kuziendeleza fikra za Kinegritudi ni Birago Diop, Leon Damas na wapigania haki za watu weusi huko Marekani kama W. E. Dubois, waandishi wa Uganda kama vile Pio Zirimu na Okot P'Bitek. Nadharia hii ina misingi mikuu minne ambapo kwanza, inasisitiza suala la ujitambuzi wa Mwafrika. Hili ni tatizo ambalo limebakia kuwa sugu katika maisha yetu kutokana na athari za ukengeushi. Pili, inapiga vita ukoloni mamboleo na hali ya wasomi wa Kiafrika kutothamini Uafrika wao. Tatu, inashutumu ukoloni kuwa ndiyo chanzo ya kudidimia kwa ujumi wa Kiafrika. Nne, inasisitiza mtindo wa kiutamaduni wa maisha(hasa maisha ya kijijini ambayo yanahusiana na kutegemea ukulima na ufugaji.

\subsection{Vitabu Teule vya Ushairi Vinavyotumika katika Makala hii}

Makala haya yameteua diwani tatu ambazo ni: Diwani ya Mnyampala ya Mathias Mnyampala ilichapishwa mwaka 1965, Mashairi ya Saadan ya Saadan Abdu Kandoro iliyochapishwa mwaka 1972 na Kivuli cha Mvumo ya Rashid Mfaume iliyochapishwa mwaka 2016. Diwani ya Mnyampala na Mashairi ya Saadam zimeteuliwa kwa sababu mashairi yaliyotumika yamegusa kwa undani ujumi wa Waafrika. Aidha, Kivuli cha Mvumo mbali na kugusia ujumi wa Kiafrika pia imeteuliwa kutokana na kigezo cha wakati hii ni kwa sababu ni diwani hii ni ya hivi karibuni.

\section{2.o. Uthibitisho wa kuwa Uwi ni Sifa ya Kiujumi wa Waafrika}

Vipengele vya ujumi wa Kiafrika vinathibitisha kuwa uwi ni sifa ya ujumi wa Kiafrika. Ufuatao ni ufafanuzi wa vipengele vya kuainisha ujumi wa Kiafrika vinavyothibitisha kuwa uwi ni sifa ya ujumi wa Kiafrika:

\subsection{Imani za Jadi}

Hii ni sifa mojawapo ya ujumi wa Kiafrika ambayo huweza kupambanuliwa kupitia uwi. Kwa mfano, kupitia vitu kama vile uchawi na sihiri, uwi hujitokeza hasa pale watu 

wanapotumia kuleta madhara kwa wengine kama vile kusababisha kifo. Tempels (1959) anaeleza kwamba Waafrika wanaamini sana katika nguvu hai na wanaamini kwamba chanzo kikuu cha nguvu hai ni miungu. Hoja hii inahalisika katika kipindi ambacho barani Afrika hakukuwa na mwingiliano wa dini za Kimagharibi. Ujumi hupatikana pale ambapo imani hizi hutumika kuleta manufaa kwa watumiaji wake. Mashairi mbalimbali yamejadili suala hili. Kwa mfano, Diwani ya Mnyampala kwenye shairi la "Binadamu Haridhiki" mshairi anasema:

Binadamu haridhiki, heri kuchunga mnyama Moyoni hatakasiki, mbeleyo hina salama Husemwa hasalifiki, wahenga waliyasema Fadhili mpe mnyama, binadamu haridhili. (uk.29)

Nukuu hiyo ya ubeti wa pili inazungumzia uwi wa binadamu katika matendo mbalimbali. Kumfadhili binadamu yaonekana kama kitu cha kupoteza muda kwa sababu malipo yake huweza kuwa madhara. Kwa mfano, baadhi ya binadamu hutumia imani za dini kuwafanyia wengine ushirikina ili wakidhi mahitaji yao fulani. Faustine (2012) anashadidia kwamba uchawi ni hali ya utumiaji wa vitu mbalimbali vilivyoko katika mazingira yetu (imani za jadi) kinyume na matumizi yake ya kawaida. Uchawi ni kitu kibaya ambacho kina sifa ya ujumi wa Waswahili. Kwa mfano, baadhi ya Waswahili huamini kuwa watu huchukuliwa nyakati za usiku wakiwa usingizini na kupelekwa shambani ili walime. Suala hili ni uwi kwa sababu watendewa hupata madhara ingawa mtenda (mchawi) hunufaika pengine kwa kupata mazao ya kutosha. Pia, kwenye diwani ya Kivuli cha Mvumo, mshairi amebainisha namna ambayo uwi ni sifa ya kiujumi kupitia suala la imani za jadi. Tunaona waganga wa jadi wanavyotumia ujumi wa Kiafrika kwa namna ya kuleta uwi. Mbiti (1969) anaeleza kuwa kazi za waganga wa jadi ni kutibu na kukinga magonjwa mbalimbali kwa binadamu, kutabiri na kukinga matatizo yanayoweza kutokea katika jamii. Waganga hawa wakati mwingine hutumia imani hizi za dini kukidhi mahitaji yao binafsi na wateja wao hali ambayo inazua taharuki na kuleta madhara kama vile vifo. Kwa mfano, shairi la "Leo", mshairi anasema:

Utaona watu wameshika marungu,

Wengine mapanga,

Kama wanawinda ngungusi,

Utasikia vishindo vya miguu!

Mara kilio!! Nakufa!! Nisaidieni!

Maskini! Albino anauawa,

Au kikongwe anatobolewa macho, 
Kwa imani za kishirikina,

Yangu macho mwanafani!

Leo imani hakuna, ndivyo nionavyo mimi. (uk. 10)

Ubeti huo wa nane unadhihirisha uwi kupitia imani za dini za Waafrika ambazo wanaziamini. Waganga wa kienyeji wamekuwa chanzo cha vifo vya albino kwa sababu watu hupewa masharti ya kuwaua albino ili wajipatie utajiri. Suala lingine ni kuhusu imani potofu ya kuwaua vikongwe kutokana na macho yao kuwa mekundu. Imani za jadi ni suala zuri katika jamii lakini ikitumika vibaya huleta uwi na uharibifu mkubwa ingawa linabakia kuwa la kiujumi kwa watumiaji wake.

Suala la uwi kama sifa ya ujumi wa Waafrika linasawiriwa kwenye diwani ya Mashairi ya Saadan. Kwa mfano, kupitia shairi la "Binadamu Tenda Wema" msanii anasema:

Japo utendwe ubaya, mlipaji lipa wema

Kwa vitendo na hekaya uwe mtu wa huruma

Uwaombee wabaya, wapate tabia njema

Binadamu tenda wema, japo utendwe ubaya (uk.18).

Ubeti huo wa nne unatoa picha pana kuwa hata kama binadamu atafanyiwa ubaya mathalan wa kisihiri, anapaswa kuendelea kufanya mema kwa sababu kwa jamii ya Waafrika husema wema hauozi. Hivyo, wanasisitiza kuwa hata kama mtu utatendewa mabaya kiasi gani hupaswi kulipa kisasi badala yake unapaswa kuendelea kulipa wema.

\subsection{Umoja}

Umoja kama kipengele cha ujumi wa Kiafrika huweza kupambanuliwa kupitia uwi. Wiredu (1980) anaeleza kuwa hali ya mshikamano na umoja miongoni mwa jamii nyingi za Kiafrika unaanzia katika familia, hadi katika jamii nzima. Hii inadhihirisha ni kwa namna gani Waswahili ambao ni jamii ya Waafrika wanavyothamini suala la umoja kwa kulikuza kuanzia ngazi ya familia. Kwa mfano, matukio ya uwi kama vile vita, njaa na vifo hufanya dhana ya umoja wa Waswahili uwe madhubuti. Hivi ni vitu vinavyoleta simanzi na huzuni kubwa ingawa vina sifa ya kiujumi kwa sababu huimarisha umoja miongoni mwa wanajamii. Kwa mfano, mashairi mbalimbali yamejadili uwi kupitia tukio la kifo. Kwenye Diwani ya Mnyampala, shairi la "Watunzi tuna huzuni”, uwi umejadiliwa mshairi anaposema: 
Kimetukata makali, na kututia huzuni,

Huzuni na udhalili, kwetu washairi nchini

Kupata pevu ajali, ya kifo chake Rubani,

Kifo cha Bwana Shaaban, kimetutia huzuni' (uk.150)

Shairi hili kupitia ubeti wa pili linaonesha uwi ambao una sifa ya kiujumi. Waafrika huthamini sana suala la umoja lakini ikumbukwe kuwa matukio yenye uwi kama vile vifo hudumisha umoja miongoni mwa wanajamii. Kwa mfano, mshairi anaposema "Watunzi tuna huzuni" kwenye kichwa cha shairi anaonesha kuwa Waswahili wamejijengea utamaduni wa kushiriki katika misiba na ikitokea sababu ya kutoshiriki basi hupata nafasi ya kuhani msiba. Katika shairi la "Watunzi tuna huzuni" inaonekana namna kifo cha Shaaban Robert kilivyohuzunisha na kuwafanya watu hususan washairi kushiriki katika msiba huo. Kimsingi, lengo la watu kushiriki katika msiba ni konesha umoja na mshikamo katika jamii na kuwafariji wafiwa katika kipindi cha majonzi.

Pia, kwenye Mashairi ya Saadan, uwi umejichomoza kupitia suala la kifo ambapo tunaona mshairi anaomboleza kifo cha mjomba wake Kalutta kwenye shairi la "Mola Muweke Peponi Mjomba wangu Kalutta”, kwenye ubeti wa pili, mshairi anasema:

Njiani ninapopita, mawazo tele moyoni, Msiba niliopata, kifo cha mwanachuoni, Kifo cha Sheikh Kalutta, kimenikaba yakini, Mola muweke peponi, mjomba wangu Kalutta” (uk.5o)

Katika ubeti huo wa pili, mshairi anamwombea kheri Kalutta kwa Mungu. Hii inaonesha kuwa kulikuwa na kiwango kikubwa cha umoja baina yake na mshairi. Umoja na ushirikiano kati ya Kandoro na Kalutta ulikuwa usiomithilika na hili linajibainisha pale tunapoona mashairi mbalimbali waliyokuwa wanapokezana. Kwa mfano, kwenye diwani hii ya Kandoro kuna mashairi na tenzi mbalimbali zilizotungwa na Kalutta kama namna ya kuwasiliana na Kandoro. Miongoni mwa mashari hayo ni; "Saadan" (uk.5), "Niliijengaje Nyumba" (uk.12), na "Waadhi wa Uongofu kwa Mwenyezi Mungu" (uk.37). Kifo cha Kalutta kinatukumbusha kudumisha umoja miongoni mwetu. Kwa mfano, Saadan alimwandikia Kalutta shairi la "Penye Tabu na Mashaka" anasema:

Kweli Napata vituko, mkweli alotamka

Nimeona badiliko, viumbe wamegeuka, Uchungu na sikitiko, Tabora nimeondoka, Penye tabu na mashaka, hutokea badiliko. (uk.16) 

Shairi la "Penye Tabu na Mashaka" lilitungwa kumfahamisha Kalutta taabu ya Saadan aliyoipata ya ukosefu wa fedha kutokana na uharibifu wa duka. Hii inadhihirisha dhana ya umoja hasa pale watu wanapopata matatizo hushirikishana kwa lengo la kusaidiana.

Pia, shairi lingine la Kandoro (1972) linaloitwa "Vita vya Njaa Vikali" linazungumzia uwi unaopatikana iwapo kuna janga la njaa. Kwenye ubeti wa kwanza, mshairi anasema:

Vita zote mkipima, mkituliza akili, Hata nanyi mtasema, vita vya njaa vikali, Sasa tusimame wima, kuifukuza awali, Vita vya njaa vikali, iwapo hamkulima. (uk.6o)

Dondoo hili linaonesha kuwa njaa ni tukio la kiuwi ambalo hutesa watu wengi. Mshairi anajaribu kuzungumzia suala la umoja ambalo ni kipengele muhimu kwa ujumi wa Waafrika. Kituo cha ubeti kinachomalizia na maneno "Vita vya njaa vikali, iwapo hamkulima”, kinapendekeza watu kushirikiana katika shughuli za kilimo ili wajiepushe na njaa.

Diwani ya Kivuli cha Mvumo, pia imeonesha suala la ujumi kupitia matukio ya uwi. Kwa mfano, shairi la "Msisonyane Wanangu”, mwandishi anasema:

Msikabane kooni, kuhoji ya asilini

Wanangu tafakarini, ugomvi jambo la soni

Kaida njema ni nduni, za kuwapa ahueni

Ishini kwa ihsani, msisonyane wanangu (uk. 8)

Nukuu hiyo kutoka ubeti wa kumi na tisa inaonesha baba aliye katika hali ya kuaga dunia akiwaasa wanawe kuwa na umoja. Hali ya umahututi wa baba huyo ni uwi ambao unashadidia umoja kuwa ni sifa ya kiujumi wa Waafrika. Wazazi wengi wa Kiafrika huwa na hulka na kutoa wosia kabla ya kuaga dunia hasa katika hali ya umahututi kwa kukazia mambo mbalimbali ikiwemo suala la umoja ambalo ni sifa ya ujumi wa Waafrika.

\subsection{Utii na Adabu}

Waafrika wanatumia utii na adabu kama kigezo cha kuupima ujumi. Uwi huweza kutumika kama nyenzo ya kuwasilisha ujumi huu wa Waswahili. Kwa mfano kwenye Diwani ya Mnyampala, suala hili limejadiliwa kwenye shairi la "Heri wenye Sura Mbaya”, msanii anasema: 
Heri mwenye sura mbaya, bora asiwe ayari

Kwa mazuri na mabaya, ajue ipi Johari

Ashinde hizo hekaya, kuyavuka makufari

Sura na iweje nzuri, hutompenda kayaya (uk.8)

Uwi kama sifa ya kiujumi katika shairi hili umeongelewa kwa kuonesha ubaya wa sura ya mtu kuwa si kigezo cha kupima tajriba yake. Mtu anaweza kuwa na sura mbaya lakini akawa mzuri wa tabia. Ujumi hujidhihirisha pale tunapoona ni heri kuwa na ubaya wa sura kuliko ule wa tabia. Ubaya wa tabia huweza kuonekana kupitia tabia kama vile uongo, ukahaba na wizi. Tabia njema huonesha utii na adabu kitu ambacho kinapewa thamani kubwa ya kiujumi kwa Waaafrika. Hivyo, sura mbaya ina heri kuliko tabia mbaya. Waafrika hupenda kumwona mtu mwenye tabia njema kuliko uzuri wa sura.

\subsection{Ushababi}

Ushababi ni hali ya mtu kuwa jasiri na kuweza kutenda vitu visivyo vya kawaida. Madembwe (2011) na Williady (2013) wanaeleza kuwa ujasiri ni sifa muhimu na hazina kubwa katika utamaduni wa maisha ya Waafrika. Ushababi ni kitu muhimu kinachodokeza ujumi wa Kiafrika ambacho hudokeza umri wa kijana mwenye nguvu, uwezo wa kufanya mambo kishupavu na uwezo wa kuzaa. Hiki ni kipengele cha ujumi wa Waafrika kinachodhihirisha kuwa uwi ni sifa ya ujumi wa Kiafrika. Kwa mfano, kwenye diwani ya Kivuli cha mvumo, "shairi la ugomvi wa wanandoa", ubeti wa tatu mshairi anasema:

Walizimwaga hongera, watu waliofurika,

Makofi ya jorajora, vicheko vilisikika,

Mnatiana bakora, penzi limeshachujika

Ugomvi wa wanandoa, unaathiri watoto (uk. 32)

Mshairi katika ubeti wa tatu anaonesha misukosuko inayowakumba wanandoa na kama ilivyozoeleka kwa jamii za Waswahili, kijana wa kiume kuoa na kijana wa kike kuolewa ni kitendo cha ushababi. Hivyo, licha ya maisha ya ndoa kuwa na uwi wa namna mbalimbali bado suala la kuoa/ kuolewa hudhihirisha ujumi wa Waswahili. Watu wasipooa/ kuolewa ilhali wana umri mkubwa huonekana kuwa na kasoro kwa sababu hukiuka idili zilizopo katika jamii zao. 

Aidha, kwenye Mashairi ya Saadan, shairi la "Ukitaka Upendeze Uhisabiwe ni Mume" suala la uwi kama sifa ya kiujumi wa Waafrika limejitokeza kupitia ndoa ambacho ni kipengele cha ushababi. Simchimba (2012) anaeleza kuwa ndoa ni jambo la msingi katika maisha ya binadamu. Hii ni hatua muhimu kwa jamii za Waafrika kwa sababu huonesha ushababi. Mshairi kwenye ubeti wa 13 anasema:

Ukioa ndugu yangu, yote uyaelekeze, Ndoa za sasa ni chungu, kula zito ulimeze Usiwe na dungu dungu, mwache lake afanze, Ukitaka upendeze, uhisabiwe ni mume. (uk. 115)

Ubeti huu bado unasisitiza uwi ulioko kwenye maisha ya ndoa. Kuoa/ kuolewa ni kitendo cha kuthibitisha ushababi wa Waswahili lakini ndoa zimekuwa na matatizo mengi. Kwa mfano, usaliti ni kitu kinachoharibu ndoa nyingi za siku hizi.

Shairi la "Mzigo Nimelemewa" la Saadan linathibitisha kuwa uwi ni sifa ya ujumi wa Kiafrika, mshairi anasema:

Vijana wasikilivu, wenye ari na takuwa

Mmezima ushupavu, wa binti mrubuniwa, Mmenitwika undovu, name ninauchukuwa, Mzigo nimelemewa, ninajitia undovu (uk. 102)

Ubeti huu wa tisa unaonesha kuwa vijana ambao ni mashababi huweza kutumia nguvu zao kutenda mambo yaliyo kinyume na idili za Kiafrika. Kwa mfano, vijana wa kiume huweza kutumia suala la kuwa shababi kwa namna ya kuwa na mahusiano ya kingono na wanawake wengi. Katika jamii ya sasa vijana hao hufanya hivyo ili tu wasifiwe na vijana wenzao kwamba wao ni shupavu. Suala hili huleta uwi kwa sababu ni tabia hatarishi.

\subsection{Lugha}

Lugha ni chombo muhimu cha mawasiliano kati ya mtu na mtu, mtu na jamii, au fanani na

hadhira yake. Hii ni ishara ya mawasiliano baina ya wanajamii na ndiyo nyenzo ya utambulisho wa maisha ya jamii. Lugha hutambulisha kaida au mfumo fulani wa maisha ya jamii fulani. Ujumi wa Waafrika hupatikana pale ambapo lugha kama ishara huakisi uhalisi wa maisha ya jamii hiyo. Diwani teule zimefumwa kwa kutumia lugha inafungamana na imani, mitazamo, na falsafa ya Waafrika. Pia, kuna kauli za asili 
zinazofungamana na maisha halisi ya wanajamii. Kwa mfano, methali na misemo hufungamana na itikadi za kiimani za Waafrika.

Kwa mfano, katika Shairi la "Muenda Omo na Tezi” katika Diwani ya Mnyampala, methali zimetumika. Mshairi anasema:

Achumaye juani, kwa methali ya walezi,

Hwenda lia kivulini, falau wali kwa nazi,

Na ndipo pumzikoni, kuyaacha matembezi,

Muenda omo na tezi, marejeo ni ngamani (uk.20)

Mishororo miwili ya mwanzo ya ubeti hapo juu imeonesha methali ya mchumia juani hulia kivulini. Methali hii inaonesha thamani ya kiujumi kwa maana kuwa Waafrika hupata taabu katika harakati za kujipatia kipato. Suala la kukumbana na changamoto mbalimbali zinazoleta madhila katika harakati za kufanikiwa ni kitu kibaya ingawa baadaye huonesha ujumi kwa kuleta matokeo mazuri. Aidha methali nyingine inayodhihirisha kuwa uwi ni sifa ya ujumi wa Kiafrika ni ile inayopatikana katika mshororo wa mwisho wa ubeti tajwa hapo juu ni "Muenda omo na tezi, marejeo ni ngamani." Kwa mujibu wa Wamitila (2001), methali hii ina maana kuwa mtu huthamini kwao hata kama ni kubaya namna gani hivyo hata akisafiri na kwenda sehemu za mbali na nyumbani hukumbuka kurejea kwao.

\section{3.o Hitimisho}

Hivyo basi, makala yamebaini kuwa uwi ni sifa ya ujumi wa Kiafrika unaosawiriwa kupitia kazi mbalimbali za kifasihi. Usawiri huo unajibainisha kupitia vipengele anuai kama vile imani za jadi, umoja, utii na adabu, ushababi na lugha. Vipengele hivi vinasawirika vyema kwa kutumia Nadharia ya Unegritudi ambayo imefungamana na ujumi wa Kiafrika. Aidha, makala inatoa rai kwa wahakiki na wadau mbalimbali wa fasihi kuangalia uwi kama kipengele muhimu kwenye taaluma ya ujumi. Mawazo ya kuwa ujumi huhusisha uzuri pekee hayahalisiki kwa sasa na hayapaswi kuendelea kupewa kipaumbele. Pia, ikumbukwe kuwa uwi ni suala linalokanganya katika kulielezea kwa sababu uwi kwa mtu mmoja huweza kuwa uzuri kwa mtu mwingine. Makala haya yametatua tatizo hili kwa kuonesha kuwa uwi ni suala lisiloepukika kwa sababu ni kinyume cha uzuri. Zingatio ni kwa kwamba, uwi katika muktadha wa ujumi hutazamwa kwa namna inayoleta uthamani.

\section{Marejeleo}


Badru, Z. (2015). Taswira na Ubainishaji wa Mabadiliko ya Kiujumi katika Vitendawili: Mfano $\quad$ wa Vitendawili: Mfano wa Vitendawili vya Kiswahili. Tasnifu ya Shahada ya Uzamivu katika Fasihi ya Kiswahili, Chuo Kikuu cha Dodoma, (Haijachapishwa).

Darwin, C. (1982). The Expression of the Emotions in Man and Animals. England: John Murray's publisher.

Faustine, S. (2012). Matumizi ya Mtindo wa Uhalisiamazingaombwe katika Riwaya Teule za Euphrase Kezilahabi: Dhina na Athari zake kwa Hadhira. Tasnifu ya Shahada ya Uzamili katika Fasihi ya Kiswahili, Chuo Kikuu cha Dodoma, (Haijachapishwa).

Kandoro, S.A. (1972). Mashairi ya Saadan. Dar es Salaam: Shirika la Magazeti ya Chama LTD.

Laswai, N. A. (2015). Ujumi katika Muziki wa Dansi: Mfano kutoka katika Nyimbo Teule za Marijani Rajabu na Ally Choki. Tasnifu ya Shahada ya Uzamili katika Fasihi ya Kiswahili, Chuo Kikuu cha Dodoma, (Haijachapishwa).

Luhwago, N. (2013). Ujumi katika Fasihi Simulizi: Mfano kutoka katika Hadithi za Wahehe. Tasnifu ya Shahada ya Uzamili katika Fasihi ya Kiswahili, Chuo Kikuu cha Dodoma, (Haijachapishwa).

Makumba, M. M. (2007). An Introduction to African Philosophy. Limuru: Paulines Publications Africa.

Mbiti, J. S. (1969). African Religion and Philosophy. London: Morrison and Gibb Ltd. Madembwe, C. J. (2011). Nafasi ya Kejeli na Dhihaka katika Tamthiliya za Penina Muhando. Tasnifu ya Shahada ya Uzamili katika Fasihi ya Kiswahili, Chuo Kikuu cha Dodoma, (Haijachapishwa).

Mfaume, R. R. Y. (2016). Malenga wa Millenia (3): Kivuli cha Mvumo. Dar es Salaam: Tanzania Adventist Press.

Mnyampala, M. (1965). Diwani ya Mnyampala. Dar es Salaam: Eastern Africa Publications.

Mushengyezi, A. (2003). Twentieth Century Literary Theory. Kampala: Mukono Publishing Company.

Oruka, H. O. (1991). Sage Philosophy: Indigenous Thinkers and Modern Debate on African Philosophy. Nairobi: Shirikon Publishers.

Oxford (2001). Concise Oxford English Dictionary. United Kingdom: Oxford University Press. 
Ponera, A. (2014). Utangulizi wa Fasihi Linganishi. Dar es Salaam: Karl Jamer Publishers.

Simchimba, E. (2012). Falsafa ya Kiafrika katika Methali za Kiswahili. Tasnifu ya Shahada ya Uzamili katika Fasihi ya Kiswahili, Chuo Kikuu cha Dodoma, (Haijachapishwa).

Tempels, P. (1959). The Bantu Philosophy. Paris: Presence Africaine.

TUKI (2013). Kamusi ya Kiswahili Sanifu. Dar es Salaam: Oxford University Press.

TUKI (2014). Kamusi ya Kiswahili Sanifu. Dar es Salaam: Oxford University Press.

Williady, H. (2013). Mabadiliko kutoka U-Aristotle kwenda Ujadi wa Kiafrika: Mifano kutoka Tamthiliya Teule za E. Hussein na P. Muhando. Tasnifu ya Shahada ya Uzamili katika Fasihi ya Kiswahili, Chuo Kikuu cha Dodoma, (Haijachapishwa).

Wamitila, K. W. (2001). Kamusi ya Methali. Nairobi: Longhorn Publishers.

Wamitila, K.W. (2002).Uhakiki wa Fasihi, Misingi na Vipengele Vyake. Nairobi: Phoenix Publishers.

Wiredu, K. (1980). Philosophy and African Culture. London: Cambridge University Press.

Zirimu, P. (1971). Black Aesthetics. Nairobi: East African Literature Bureau. 